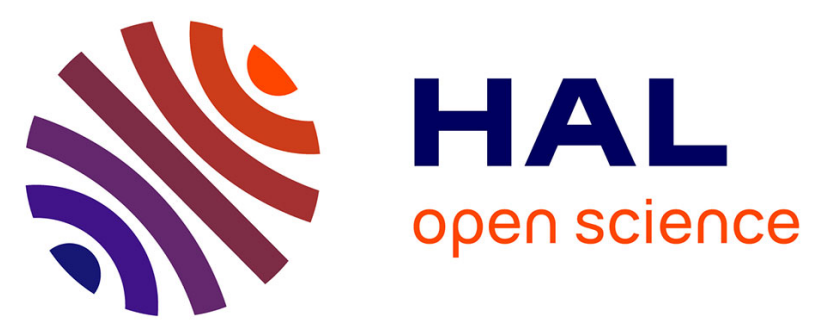

\title{
Optimal Design of Cable-Driven Parallel Robots for Large Industrial Structures
}

Lorenzo Gagliardini, Stéphane Caro, Marc Gouttefarde, Philippe Wenger, Alexis Girin

\section{- To cite this version:}

Lorenzo Gagliardini, Stéphane Caro, Marc Gouttefarde, Philippe Wenger, Alexis Girin. Optimal Design of Cable-Driven Parallel Robots for Large Industrial Structures. ICRA: International Conference on Robotics and Automation, May 2014, Hong-Kong, China. pp.5744-5749, 10.1109/ICRA.2014.6907703 . lirmm-01221401

\section{HAL Id: lirmm-01221401 \\ https://hal-lirmm.ccsd.cnrs.fr/lirmm-01221401}

Submitted on 1 Dec 2018

HAL is a multi-disciplinary open access archive for the deposit and dissemination of scientific research documents, whether they are published or not. The documents may come from teaching and research institutions in France or abroad, or from public or private research centers.
L'archive ouverte pluridisciplinaire HAL, est destinée au dépôt et à la diffusion de documents scientifiques de niveau recherche, publiés ou non, émanant des établissements d'enseignement et de recherche français ou étrangers, des laboratoires publics ou privés. 


\title{
Optimal Design of Cable-Driven Parallel Robots for Large Industrial Structures
}

\author{
Lorenzo Gagliardini ${ }^{1}$, Stéphane Caro $^{2}$, Marc Gouttefarde ${ }^{3}$, Philippe Wenger ${ }^{2}$ and Alexis Girin ${ }^{1}$
}

\begin{abstract}
This paper presents the preliminary studies dedicated to the design of cable-driven parallel robots (CDPRs) for industrial purposes. The goal is to transport the proper tools around a jacket, an offshore structure supporting a wind turbine, in order to perform painting and sandblasting tasks. In this paper, a simplified case study consisting of a structure composed of four tubes is investigated. A fully constrained CDPR and a suspended CDPR are studied. The design problems of the CDPRs at hand are formulated as optimization problems. They aim at determining the locations of the base anchor points of the cables that minimize the size of the CDPR, while satisfying a set of constraints. Those constraints guarantee that the moving platform can support the external wrenches and that there is no interference between the cables and between the cables and the environment, all along the path to be followed by the moving platform.
\end{abstract}

\section{INTRODUCTION}

Nowadays part of the worldwide energy production comes from renewable sources: offshore wind turbines represent a leading technology in the renewable energy sector [1]. They can be installed in the sea through different supports, like jackets (offshore fixed structures, as illustrated in Fig. 1). Due to their shape and size, the fabrication of jackets is complicated and requires non standard procedures. The study of efficient technological processes aims at producing these structures in series and at limiting the risks for operators. In this context, the IRT Jules Verne is cooperating with STX Europe to investigate and develop a Cable Driven Parallel Robot (CDPR) in order to perform painting and sandblasting operations on jackets.

CDPRs can represent an appropriate technological solution for the considered tasks. Indeed, CDPR are able to cover wide spaces and their payload to weight ratio is usually very high. They are relatively cheap and can be designed in such a way to be reconfigurable and therefore adapted to different tasks.

Although no CDPR has already been designed for the application considered in this paper, research works have already been performed in similar sectors, like the naval, aeronautic and civil engineering ones. Albus et al. developed, at

This work is supported by the IRT Jules Verne, Bouguenais, France (Project CAROCA, Evaluation des CApacités de la RObotique à CÂbles dans un contexte industriel)

${ }^{1}$ L. Gagliardini and A. Girin are with Robotics Team, IRT Jules Verne, Chemin du Chaffault, 44340, Bouguenais, France, \{lorenzo.gagliardini, alexis.girin\}@irt-jules-verne.fr

${ }^{2} \mathrm{~S}$. Caro and P. Wenger are with CNRS-IRCCyN, 1, rue de la Noë, 44321, Nantes Cedex 03, France, \{Stephane.Caro, Philippe.Wenger\} eirccyn.ec-nantes.fr

${ }^{3} \mathrm{M}$. Gouttefarde is with the Laboratoire d'Informatique, de Robotique et de Micro-électronique de Montpellier (LIRMM-CNRS-UM2), 161, rue Ada, 34392, Montpellier Cedex 05, France, marc.gouttefarde@lirmm. fr

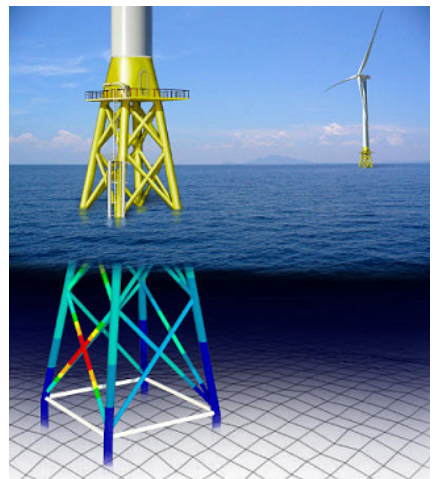

Fig. 1. Offshore wind turbine jacket, courtesy of STX Europe.

the beginning of the 90s, the NIST RoboCrane [2], a CDPR able to perform several industrial operations, like painting of military aeroplanes and displacement of heavy payloads. In 2001 Holland and Cannon filed the patent US6826452 B1 [3]. They proposed a robotic system composed of four cranes to be employed as a CDPR for cargo transportation. Currently, several research institutes and companies are involved in the framework of the European Project CableBOT that aims at developing modular and reconfigurable CDPRs able to perform different operations during the fabrication of largescale structures [4]. CDPRs can be employed also for other large workspace applications, e.g. the broadcasting of sport events [5], [6] and rescue operations [7].

This paper presents a preliminary design study of a CDPR transporting the tools necessary to perform painting and sandblasting operations on a jacket. The designs of a fully constrained CDPR and a suspended CDPR are investigated. Due to the complexity of a real jacket, the analysis is applied on a simplified structure made up of four tubes, as illustrated in Fig. 2. This context represents a new challenge since it introduces several constraints into the design problem. Due to the cluttered industrial environment, interferences between the cables and the environment need to be taken into account. The cable tensions and the positioning precision of the CDPR are constrained as well. In this paper, the design problem is formulated as an optimization problem, aiming at minimizing the size of the CDPR.

The paper is organized as follows: Section II describes the industrial context and the problem formulation. Section III introduces the CDPR models. Section IV presents the design of a fully constrained CDPR for the given case study. Section V describes the design of a suspended CDPR. Section VI concludes the paper and presents our future work. 


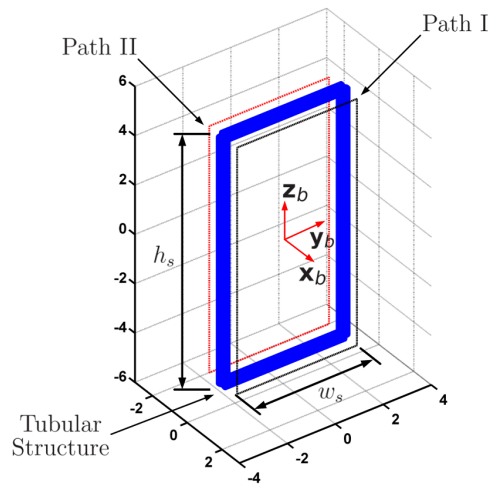

Fig. 2. Description of the task environment.

\section{PROBLEM DESCRIPTION}

A jacket is an offshore structure composed of several tubes as shown in Fig. 1. One jacket has already been realized by STX Europe. It has a base of $30 \mathrm{~m} \times 30 \mathrm{~m}$, an height of $60 \mathrm{~m}$ and a weight of $1000 \mathrm{~T}$. The upper part of the jacket, which remains outside the water, is $20 \mathrm{~m}$ high. This part should be painted and sandblasted, to be protected from oxidation. STX Europe planned to automatize part of the industrial process in order to produce several units per year.

The technical solution proposed in this paper consists in painting and sandblasting the structure by means of proper tools mounted on a CDPR platform: the Centre of Mass (CoM) of the CDPR follows a path along the jacket tubes approaching the tools to the jacket itself. When the CDPR is in the correct pose, the tools can perform the required tasks.

Due to the complexity of the problem, a simplified case study is introduced. Painting and sandblasting operations have to be performed on a four-bar tubular rigid structure. The structure is centered in an absolute reference frame, $\mathscr{F}_{b}$, of origin $O_{b}$ and axes $\mathbf{x}_{b}, \mathbf{y}_{b}, \mathbf{z}_{b}$, as shown in Fig. 2. It has an height, $h_{s}$, of $10 \mathrm{~m}$ and a width, $w_{s}$, of $5 \mathrm{~m}$. The diameter of the tube, $\phi_{s}$, is equal to $50 \mathrm{~cm}$.

The design problem can be summarized as follows. The CDPR cable exit point positions have to be determined. The CDPR should be able to follow a proper path to let the embarked painting and sandblasting tools perform the required operations along one side of the four-tube structure. The other side can be painted by a similar CDPR. Both the paths are illustrated in Fig. 2. They are $80 \mathrm{~cm}$ far from the structure. The CDPR should avoid cable interferences and collisions between the cables and the environment (here, the four-bar tubular structure). The cable tensions must respect given limits. The CDPR must be fully constrained during the operations, or at least in static equilibrium (in the case of a suspended design). The CDPR should be as compact as possible.

\section{GEOMETRIC AND STATIC ROBOT MODELS}

A CDPR is composed of a mobile platform, a support structure (the base) and $m$ cables. Two reference frames are considered: the fixed base frame, $\mathscr{F}_{b}$, presented in Section II, and a platform frame, $\mathscr{F}_{p}$, of origin $O_{p}$ and axes $\mathbf{x}_{p}, \mathbf{y}_{p}, \mathbf{z}_{p}$.

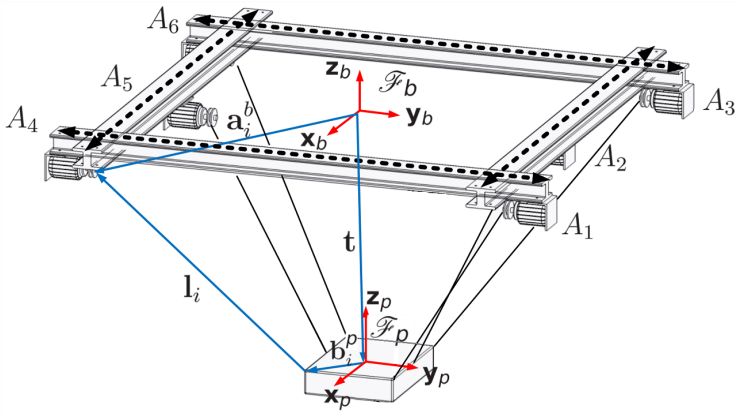

Fig. 3. Cable driven robot geometry. Example of a suspended CDPR. The same geometric description can be applied to a fully constrained CDPR.

The exit point of the $i$-th cable on the base, $A_{i}$, is described, with respect to $\mathscr{F}_{b}$, through the Cartesian coordinate vector $\mathbf{a}_{i}^{b}, i=1, \ldots, m$. The platform connection point of the $i$-th cable, $B_{i}$, is defined, with respect to $\mathscr{F}_{b}$, by the Cartesian coordinate vector $\mathbf{b}_{i}^{b}, i=1, \ldots, m$; the same vector, expressed in $\mathscr{F}_{p}$, is represented by $\mathbf{b}_{i}^{p}, i=1, \ldots, m$. The moving platform pose, $\mathbf{p}$, expressed in $\mathscr{F}_{b}$, is composed of the Cartesian coordinate vector of the CoM position, $\mathbf{t}$, and the orientation angle vector $\Phi=[\phi, \theta, \psi]^{\mathrm{T}}$, described through the Euler Angles $\phi, \theta$ and $\psi$ around $\mathbf{z}_{b}, \mathbf{x}_{b}$ and $\mathbf{y}_{b}$, respectively.

As illustrated in Fig. 3, the length of the $i$-th cable, between points $A_{i}$ and $B_{i}$, is the norm of the vector $\mathbf{l}_{i}$ expressed in $\mathscr{F}_{b}$ as:

$$
\mathbf{l}_{i}=\mathbf{a}_{i}^{b}-\mathbf{t}-\mathbf{R} \mathbf{b}_{i}^{p} \quad i=1, \ldots, m
$$

where $\mathbf{R}$ denotes the orientation matrix of the platform:

$$
\begin{aligned}
\mathbf{R} & =\mathbf{R}_{z}(\phi) \mathbf{R}_{x}(\theta) \mathbf{R}_{y}(\psi)= \\
& =\left[\begin{array}{ccc}
c \phi c \psi-s \phi s \theta s \psi & -s \phi c \theta & c \phi s \psi+s \phi s \theta c \psi \\
s \phi c \psi+c \phi s \theta s \psi & c \phi c \theta & s \phi s \psi-c \phi s \theta c \psi \\
-c \theta s \psi & s \theta & c \theta c \psi
\end{array}\right]
\end{aligned}
$$

The unit vector of the $i$-th cable, $\mathbf{d}_{i}$, expressed in $\mathscr{F}_{b}$, is equal to:

$$
\mathbf{d}_{i}=\frac{\mathbf{l}_{i}}{\left\|\mathbf{l}_{i}\right\|_{2}} \quad i=1, \ldots, m
$$

The CDPR static model is represented by the following equilibrium equation [8]

$$
\mathbf{W} \boldsymbol{\tau}+\mathbf{w}_{e}=0
$$

$\boldsymbol{\tau}=\left[\tau_{1}, \ldots, \tau_{m}\right]^{\mathrm{T}}$ denotes the cable tension vector. Due to the non-rigid nature of the cables, tensions must be non-negative. Moreover, they have to remain smaller than the maximum tension value $\tau_{\max }$. $\mathbf{W}$ denotes the wrench matrix composed of the wrenches $\mathbf{w}_{i}$ exerted by the cables on the platform at point $O_{p}$, namely,

$$
\mathbf{W}=\left[\begin{array}{cccc}
\mathbf{d}_{1} & \mathbf{d}_{2} & \ldots & \mathbf{d}_{m} \\
\mathbf{R} \mathbf{b}_{1}^{p} \times \mathbf{d}_{1} & \mathbf{R b}_{2}^{p} \times \mathbf{d}_{2} & \ldots & \mathbf{R b}_{m}^{p} \times \mathbf{d}_{m}
\end{array}\right]
$$

$\mathbf{w}_{e}$ represents the external wrench acting on the platform:

$$
\mathbf{w}_{e}=[\mathbf{f}, \mathbf{m}]^{\mathrm{T}}=\left[f_{x}, f_{y}, f_{z}, m_{x}, m_{y}, m_{z}\right]^{\mathrm{T}}
$$


Its components are supposed to be bounded.

$$
\begin{gathered}
f_{\min } \leq f_{x}, f_{y}, f_{z} \leq f_{\max } \\
m_{\min } \leq m_{x}, m_{y}, m_{z} \leq m_{\max }
\end{gathered}
$$

When the number $m$ of cables is greater than 6 , the system of equations (4) is underdetermined and the solution can be expressed as:

$$
\boldsymbol{\tau}=\boldsymbol{\tau}_{n}+\boldsymbol{\tau}_{0}=\mathbf{W}^{\dagger} \mathbf{w}_{e}+\lambda \mathbf{n} \quad \tau_{n} \geq \mathbf{0}
$$

$\mathbf{W}^{\dagger}$ denotes the Moore-Penrose generalized inverse of $\mathbf{W}$, $\lambda \in \mathbb{R}$ and $\mathbf{n}$ is a vector in the null space of $\mathbf{W}$ [8].

\section{FULLY CONSTRAINED CDPR DESIGN}

The first design problem aims at minimizing the size of a fully constrained CDPR intended to paint a side of the tubular structure. To perform this operation, the CDPR CoM should follow the Path I shown in Fig. 2. The other side can be painted by a symmetrically placed CDPR following Path II. Each path is defined by a discretized curve $\mathscr{P}$, composed of 600 equidistant points $P$. The CDPR static equilibrium is an important issue to take into consideration since the cables cannot push the platform. Therefore, the CDPR equilibrium can be satisfied only through positive cable tensions. The required robot accuracy is also considered, as well as the collisions between cables and between cables and the tubular structure. These constraints must be verified for all points $P \in \mathscr{P}$.

The optimization is performed on a CDPR with eight cables, $m=8$, which corresponds to the minimum even number of cables for a fully constrained CDPR [8]. This choice should maintain the design of the CDPR as simple and cheap as possible. Steel cables are considered. They are characterized by the following properties: diameter, $\phi_{c}$, of $0.4 \mathrm{~cm}$, Young Modulus equal to $100 \mathrm{GPa}$, elastic coefficient $k$ of $1,256 \times 10^{6} \mathrm{~N} / \mathrm{m}$ and tension limit, $\tau_{\max }$, equal to $11650 \mathrm{~N}$. In order to simplify the problem, the layout of points $B_{i}, i=1, \ldots, 8$ is predefined. Points $B_{i}$ lie at the corners of a parallelepiped, whose width, $w_{p}$, length, $l_{p}$, and height, $h_{p}$, are equal to $40 \mathrm{~cm}, 40 \mathrm{~cm}$ and $20 \mathrm{~cm}$, respectively.

\section{A. Design Variables}

The decision variables of the optimization problem are represented by the position vectors of points $A_{i}$. To reduce the number of variables, the points $A_{i}$ have been located at the vertices of parallelepiped whose edges are parallel to the axes $\mathbf{x}_{b}, \mathbf{y}_{b}$ and $\mathbf{z}_{b}$. Hence, four variables, $u_{x 1}, u_{x 2}, u_{y}$, $u_{z}$, are sufficient to define the coordinates of points $A_{i}$ with respect to $\mathscr{F}_{b}$, as illustrated in Fig. 4,

$$
\begin{aligned}
\mathbf{a}_{1}^{b}=\left[u_{x 1}, u_{y}, u_{z}\right]^{\mathrm{T}}, & \mathbf{a}_{2}^{b}=\left[u_{x 1}, u_{y},-u_{z}\right]^{\mathrm{T}} \\
\mathbf{a}_{3}^{b}=\left[u_{x 2}, u_{y}, u_{z}\right]^{\mathrm{T}}, & \mathbf{a}_{4}^{b}=\left[u_{x 2}, u_{y},-u_{z}\right]^{\mathrm{T}} \\
\mathbf{a}_{5}^{b}=\left[u_{x 2},-u_{y}, u_{z}\right]^{\mathrm{T}}, & \mathbf{a}_{6}^{b}=\left[u_{x 2},-u_{y},-u_{z}\right]^{\mathrm{T}} \\
\mathbf{a}_{7}^{b}=\left[u_{x 1},-u_{y}, u_{z}\right]^{\mathrm{T}}, & \mathbf{a}_{8}^{b}=\left[u_{x 1},-u_{y},-u_{z}\right]^{\mathrm{T}}
\end{aligned}
$$

The boundaries of the design variables are defined as follows:

$$
\begin{array}{ll}
0.8 \mathrm{~m} \leq u_{x 1} \leq 3.5 \mathrm{~m}, & 0 \mathrm{~m} \leq u_{x 2} \leq 0.8 \mathrm{~m} \\
2.5 \mathrm{~m} \leq u_{y} \leq 5 \mathrm{~m}, & 5 \mathrm{~m} \leq u_{z} \leq 7.5 \mathrm{~m}
\end{array}
$$

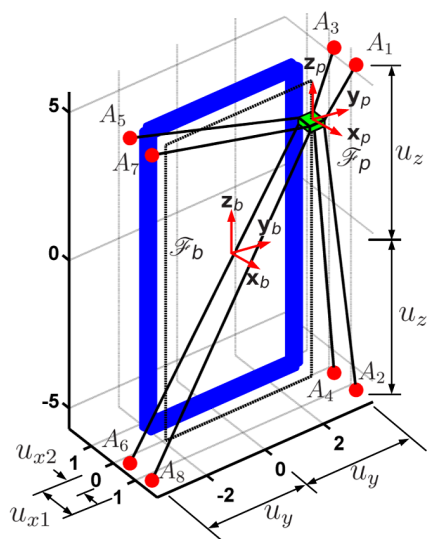

Fig. 4. Layout of points $A_{i}$ for the fully constrained CDPR.

TABLE I

REQUiRED WRENCH SET DEFINITION

\begin{tabular}{l|llllll}
\hline \hline & $f_{x}$ & $f_{y}$ & $f_{z}$ & $m_{x}$ & $m_{y}$ & $m_{z}$ \\
\hline $\min$ & $-50 \mathrm{~N}$ & $-50 \mathrm{~N}$ & $-600 \mathrm{~N}$ & $-7.5 \mathrm{Nm}$ & $-7.5 \mathrm{Nm}$ & $-7.5 \mathrm{Nm}$ \\
$\max$ & $50 \mathrm{~N}$ & $50 \mathrm{~N}$ & $-550 \mathrm{~N}$ & $7.5 \mathrm{Nm}$ & $7.5 \mathrm{Nm}$ & $7.5 \mathrm{Nm}$ \\
\hline \hline
\end{tabular}

The vector gathering the design variables is denoted $\mathrm{x}$,

$$
\mathbf{x}=\left[u_{x 1}, u_{x 2}, u_{y}, u_{z}\right]
$$

whereas $\mathbf{q}$ denotes the design parameter vector,

$$
\mathbf{q}=\left[m, \tau_{\max }, \phi_{c}, l_{p}, w_{p}, h_{p}, h_{s}, w_{s}, \phi_{s}\right]
$$

\section{B. Objective Function}

The objective function, $\mathscr{V}(\mathbf{x})$, of the optimisation problem at hand is the volume of the parallelepiped whose vertices are the points $A_{i}, i=1, \ldots, 8$ :

$$
\mathscr{V}(\mathbf{x})=4\left(u_{x 1}-u_{x 2}\right) u_{y} u_{z}
$$

This objective function has been selected in order to minimize the space occupied by the CDPR inside the workshop.

\section{Constraints}

The path to be followed by the CoM of the CDPR platform should be included in the Wrench Feasible Workspace (WFW) of the robot for the latter to be in static equilibrium and controllable [9], [10]. The WFW is the set of platform poses that are wrench feasible. A pose is wrench feasible if the CDPR is able to balance any external wrench within a given set, with non-negative cable tensions lying in a given admissible interval [11]. Hence, for the pose of the moving platform to be inside the WFW, the following condition should be fulfilled,

$$
\forall \mathbf{w}_{e} \in[\mathbf{w}]_{r}, \quad \exists \boldsymbol{\tau} \in[\boldsymbol{\tau}] \quad \mid \mathbf{W} \boldsymbol{\tau}+\mathbf{w}_{e}=0
$$

where $[\mathbf{w}]_{r}$ denotes the required wrench set, representing the set of wrenches that can be applied on the CDPR platform. Its boundaries have already been defined in Eq. (7) and Eq. (8). Their values are given in Table I. $[\boldsymbol{\tau}]$ represents 
the set of admissible cable tensions, whose components are bounded as follows:

$$
0 \leq \tau_{i} \leq \tau_{\max }, \quad \forall i=1, \ldots, 8
$$

$[\mathbf{w}]_{r}$ and $[\boldsymbol{\tau}]$ are defined in different spaces, connected through the static equilibrium equation by the wrench matrix. The wrenches that can be generated by $[\tau]$, through Eq. (4), is a zonotope, called the available wrench set, $[\mathbf{w}]_{a}=$ $-\mathbf{W}[\tau]$. In order to verify the wrench feasibility of the poses belonging to Path I, the technique described in [12] and [13] has been used. The algorithm verifies that all the vertices of $[\mathbf{w}]_{r}$ are included in $[\mathbf{w}]_{a},[\mathbf{w}]_{r} \subseteq[\mathbf{w}]_{a}$. According to [12] and [13], this condition can be translated into verifying the satisfaction of a finite set of linear inequalities:

$$
\mathbf{C w} \leq \mathbf{d}, \quad \forall \mathbf{w} \in[\mathbf{w}]_{r}
$$

Moreover, cable-cable and cable-structure potential collisions are taken into account. Being given that two cables $i$ and $j$ are represented as straight line segments, a possible cable interference can be verified by computing the distance $d_{i, j}^{c c}$ between the cables $i$ and $j$. This distance $d_{i, j}^{c c}$ is calculated by means of Lumelsky's approach [14] and has to be larger than the diameter, $\phi_{c}$, of the cables, i.e.,

$$
d_{i, j}^{c c} \geq \phi_{c} \quad \forall i, j=1, \ldots, m, \quad i \neq j
$$

For $m=8$, the number of tests to be performed for each point $P$ is equal to $C_{8}^{2}=\frac{8 !}{2 ! 6 !}=28$. Given a cable $i$ and one of the four tubes $k$ of the structure, cable-structure potential collisions are tested by means of the distance $d_{i, k}^{c s}$ between the $i$-th cable and the $k$-th tube of the structure. The $i$-th cable and the $k$-th tube do not collide when $d_{i, k}^{c s}$ is greater than the sum of the cable and tube radii.

$$
d_{i, k}^{c s} \geq \frac{\left(\phi_{c}+\phi_{s}\right)}{2} \quad \forall i=1, \ldots, m, \quad \forall k=1, \ldots, n_{c}
$$

where $n_{c}=4$ is the number of tubes of the structure considered in this paper. Consequently, the number of constraints to be considered is equal to $m n_{c}=32$.

Due to operational requirements, the positioning errors $\delta t_{x}, \delta t_{y}$ and $\delta t_{z}$ of the CoM of the moving platform along the $\mathbf{x}_{b}, \mathbf{y}_{b}$ and $\mathbf{x}_{b}$ axes should be smaller than $1 \mathrm{~cm}$ :

$$
-1 \mathrm{~cm} \leq \delta t_{x}, \delta t_{y}, \delta t_{z} \leq 1 \mathrm{~cm}
$$

The orientation errors $\delta r_{x}, \delta r_{y}$ and $\delta r_{z}$ of the moving platform about $\mathbf{x}_{b}, \mathbf{y}_{b}$ and $\mathbf{z}_{b}$ axes should be smaller than $0.1 \mathrm{rad}$ :

$$
-0.1 \mathrm{rad} \leq \delta r_{x}, \delta r_{y}, \delta r_{z} \leq 0.1 \mathrm{rad}
$$

The elastostatic model of the CDPR is defined as follows:

$$
\mathbf{w}_{e x t}=\mathbf{K} \delta \mathbf{p}=\mathbf{K}\left[\begin{array}{ll}
\delta \mathbf{r}^{\mathrm{T}} & \delta \mathbf{t}^{\mathrm{T}}
\end{array}\right]^{\mathrm{T}}
$$

where $\mathbf{K}$ is the stiffness matrix of the CDPR and the vector $\delta \mathbf{p}$ denotes the moving platform pose displacement generated by an external wrench $\mathbf{w}_{\text {ext }}$ exerted on the moving-platform. Hence, vector $\delta \mathbf{p}$ can be computed as:

$$
\delta \mathbf{p}=\mathbf{K}^{-1} \mathbf{w}_{e}
$$

TABLE II

DESIGN VARIABLE AND OBJECTIVE FUNCTION VALUES OF THE OPTIMUM FULLY CONSTRAINED CDPR

\begin{tabular}{ccccc}
\hline \hline$u_{x 1}$ & $u_{x 2}$ & $u_{y}$ & $u_{z}$ & $\mathscr{V}$ \\
\hline $0.8207 \mathrm{~m}$ & $0.7807 \mathrm{~m}$ & $3.1165 \mathrm{~m}$ & $6.2461 \mathrm{~m}$ & $3.1146 \mathrm{~m}^{3}$ \\
\hline \hline
\end{tabular}

From [15], matrix $\mathbf{K}$ can be expressed as $\mathbf{K}=\mathbf{K}_{k}+\mathbf{K}_{t}$, with

$$
\begin{aligned}
\mathbf{K}_{k}= & \sum_{i=1}^{8}\left(k_{i}-\frac{\tau_{i}}{\rho_{i}}\right)\left[\begin{array}{cc}
\mathbf{d}_{i} \mathbf{d}_{i}^{\mathrm{T}} & \mathbf{d}_{i} \mathbf{d}_{i}^{\mathrm{T}} \hat{\mathbf{b}}_{i}^{b^{\mathrm{T}}} \\
\hat{\mathbf{b}}_{i}^{b^{\mathrm{T}}} \mathbf{d}_{i} \mathbf{d}_{i}^{\mathrm{T}} & \hat{\mathbf{b}}_{i}^{b} \mathbf{d}_{i} \mathbf{d}_{i}^{\mathrm{T}} \hat{\mathbf{b}}_{i}^{b}
\end{array}\right] \\
& +\sum_{i=1}^{8} \frac{\tau_{i}}{\rho_{i}}\left[\begin{array}{cc}
\mathbf{I}_{3 \times 3} & \hat{\mathbf{b}}_{i}^{b^{\mathrm{T}}} \\
\hat{\mathbf{b}}_{i}^{b} & \hat{\mathbf{b}}_{i}^{b} \hat{\mathbf{b}}_{i}^{b^{\mathrm{T}}}
\end{array}\right] \\
\mathbf{K}_{t}= & -\sum_{i=1}^{8} \tau_{i}\left[\begin{array}{ll}
\mathbf{0}_{3 \times 3} & \mathbf{0}_{3 \times 3} \\
\mathbf{0}_{3 \times 3} & \hat{\mathbf{d}}_{i} \hat{\mathbf{b}}_{i}^{b}
\end{array}\right]
\end{aligned}
$$

where $k_{i}$ denotes the $i$-th cable stiffness. $k_{i}$ is set equal to $k$. $\mathbf{I}_{3 \times 3}$ denotes the $3 \times 3$ identity matrix. $\hat{\mathbf{b}}_{\dot{t}}^{b}$ is the cross product matrix of vector $\mathbf{b}_{i}^{b}=\left[b_{i, x}^{b}, b_{i, y}^{b}, b_{i, z}^{b}\right]^{t^{t}}$ defined as:

$$
\hat{\mathbf{b}}_{i}^{b}=\left[\begin{array}{ccc}
0 & -b_{i, z}^{b} & b_{i, y}^{b} \\
b_{i, z}^{b} & 0 & -b_{i, x}^{b} \\
-b_{i, y}^{b} & b_{i, x}^{b} & 0
\end{array}\right]
$$

$\hat{\mathbf{d}}_{i}$ denotes the cross product matrix of $\mathbf{d}_{i}$. Eq. (27) should be verified with respect to the bounds defined in Eq. (24) and Eq. (25) for all the vertices of $[\mathbf{w}]_{r}$.

\section{Design Problem Formulation}

The design problem of the CDPR can be formulated as follows:

$\begin{aligned} \operatorname{mininimize} & \mathscr{V}(\mathbf{x})=4\left(u_{x 1}-u_{x 2}\right) u_{y} u_{z} \\ \text { over } & \mathbf{x}=\left[u_{x 1}, u_{x 2}, u_{y}, u_{z}\right]\end{aligned}$

subject to

$\forall P \in \mathscr{P}\left\{\begin{array}{l}\mathbf{C w} \leq \mathbf{d}, \quad \forall \mathbf{w} \in[\mathbf{w}]_{r} \\ d_{i, j}^{c c} \geq \phi_{c} \quad \forall i, j=1, \ldots, 8, \quad i \neq j \\ d_{i, k}^{c s} \geq \frac{\left(\phi_{c}+\phi_{s}\right)}{2} \quad \forall i=1, \ldots, 8, \quad \forall k=1, \ldots, 4 \\ -1 \mathrm{~cm} \leq \delta t_{x}, \delta t_{y}, \delta t_{z} \leq 1 \mathrm{~cm} \\ -0.1 \mathrm{rad} \leq \delta r_{x}, \delta r_{y}, \delta r_{z} \leq 0.1 \mathrm{rad}\end{array}\right.$

\section{E. Optimum Fully Constrained CDPR Design}

The previous optimization problem has been solved by using the GlobalSearch Algorithm developed by Zsolt et al. [16]. The optimum design is illustrated in Fig. 5. Table II provides the design variables and objective function values for the optimum fully constrained CDPR. This design is the most compact one, as visible by the short distances between the points $A_{i}$ along $\mathbf{x}_{b}$. This small distance prevents the cables from interfering and represents an interesting technical solution in terms of cable setting on the base. 


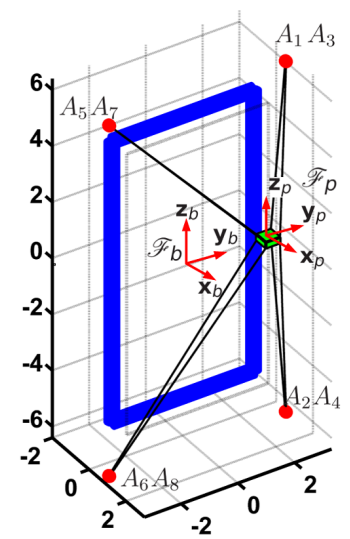

Fig. 5. Optimum design of the fully constrained CDPR. Solution of the optimization problem (31).

\section{SUSPENDED CDPR DESIGN}

The second design optimization problem aims at finding the positions of the cable exit and connection points that minimize the size of a suspended CDPR intended to perform the task detailed in Section IV. The chosen number of cables is equal to six, i.e., $m=6$. To simplify the problem, we assume that no external wrench is applied on the moving platform: only gravity is acting on the platform weighting $60 \mathrm{~kg}$. The wrench produced by the platform weight is defined by the vector $\mathbf{w}_{g}$.

\section{A. Design Variables}

Unlike in Section IV, the length $l_{p}$, the width $w_{p}$ and the height $h_{p}$ of the box-shaped moving platform are part of the variables of the design problem at hand. The configuration of points $B_{i}$ has a higher influence, in terms of static equilibrium, for suspended CDPR than for fully constrained ones. The Cartesian coordinates of points $B_{i}, i=1, \ldots, 6$ are expressed in the moving platform frame as follows:

$$
\begin{aligned}
\mathbf{b}_{1}^{p} & =\left[\frac{l_{p}}{2}, \frac{w_{p}}{2}, \frac{h_{p}}{2}\right]^{\mathrm{T}}, & \mathbf{b}_{2}^{p} & =\left[0, \frac{w_{p}}{2}, \frac{-h_{p}}{2}\right]^{\mathrm{T}} \\
\mathbf{b}_{3}^{p} & =\left[\frac{-l_{p}}{2}, \frac{w_{p}}{2}, \frac{h_{p}}{2}\right]^{\mathrm{T}}, & \mathbf{b}_{4}^{p} & =\left[\frac{-l_{p}}{2}, \frac{-w_{p}}{2}, \frac{h_{p}}{2}\right]^{\mathrm{T}} \\
\mathbf{b}_{5}^{p} & =\left[0, \frac{-w_{p}}{2}, \frac{-h_{p}}{2}\right]^{\mathrm{T}}, & \mathbf{b}_{6}^{p} & =\left[\frac{l_{p}}{2}, \frac{-w_{p}}{2}, \frac{h_{p}}{2}\right]^{\mathrm{T}}
\end{aligned}
$$

The layout of points $B_{i}$ is shown in Fig. 6. The different positions of points $B_{i}$ along $\mathbf{z}_{p}$ aim at balancing the external wrenches. The Cartesian coordinates of points $A_{i}$, illustrated in Fig. 6, are defined in terms of variables $u_{x 1}, u_{x 2}, u_{y}, u_{z}$, as:

$$
\begin{aligned}
\mathbf{a}_{1}^{b}=\left[u_{x 1}, u_{y}, u_{z}\right]^{\mathrm{T}}, & \mathbf{a}_{2}^{b}=\left[u_{x 12}, u_{y}, u_{z}\right]^{\mathrm{T}} \\
\mathbf{a}_{3}^{b}=\left[u_{x 2}, u_{y}, u_{z}\right]^{\mathrm{T}}, & \mathbf{a}_{4}^{b}=\left[u_{x 2},-u_{y}, u_{z}\right]^{\mathrm{T}} \\
\mathbf{a}_{5}^{b}=\left[u_{x 12},-u_{y}, u_{z}\right]^{\mathrm{T}}, & \mathbf{a}_{6}^{b}=\left[u_{x 1},-u_{y}, u_{z}\right]^{\mathrm{T}}
\end{aligned}
$$

where $u_{x 12}=\left(u_{x 1}+u_{x 2}\right) / 2$.

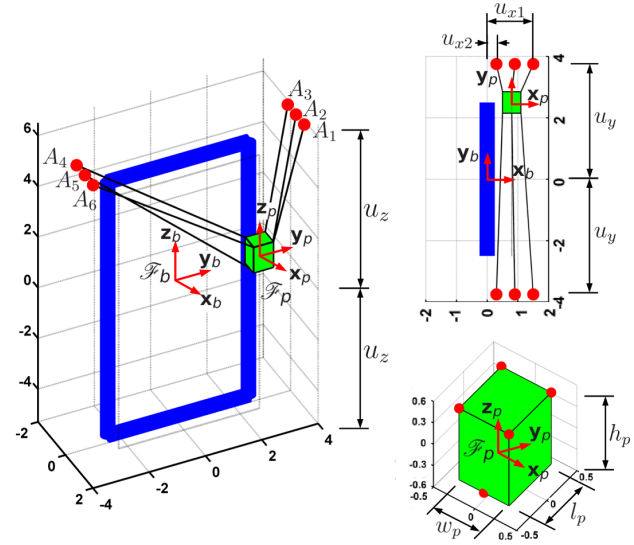

Fig. 6. Layouts of points $A_{i}$ and $B_{i}$ for the suspended CDPR.

The bounds on the design variables $u_{x 1}, u_{x 2}, u_{y}, u_{z}$ are defined in Eq. (14) and Eq. (15). The bounds on variables $l_{p}, w_{p}$ and $h_{p}$ are the following:

$$
0.3 \mathrm{~m} \leq l_{p}, w_{p}, h_{p} \leq 0.6 \mathrm{~m}
$$

The design variables are collected in the vector $\mathbf{x}$ :

$$
\mathbf{x}=\left[u_{x 1}, u_{x 2}, u_{y}, u_{z}, l_{p}, w_{p}, h_{p}\right]
$$

The design parameter vector $\mathbf{q}$ is defined as:

$$
\mathbf{q}=\left[m, \tau_{\max }, \phi_{c}, h_{s}, w_{s}, \phi_{s}\right]
$$

\section{B. Design Problem Formulation}

The objective function of the optimization problem is defined by Eq. (18). The constraints provided in Sec. IV-C are still valid. Hence, the design problem of the suspended CDPR can be formulated as follows:

$$
\begin{aligned}
\operatorname{mininimize} & \mathscr{V}(\mathbf{x})=4\left(u_{x 1}-u_{x 2}\right) u_{y} u_{z} \\
\text { over } & \mathbf{x}=\left[u_{x 1}, u_{x 2}, u_{y}, u_{z}, l_{p}, w_{p}, h_{p}\right]
\end{aligned}
$$

subject to

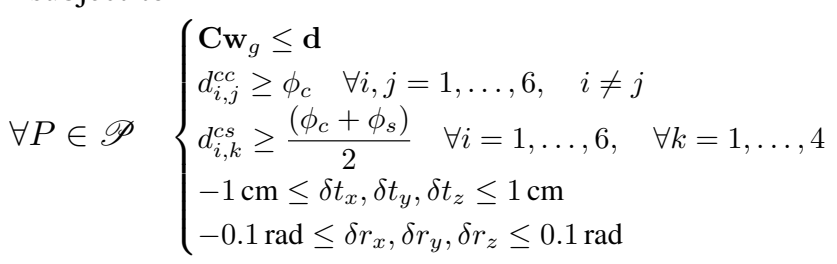

\section{Optimum Suspended CDPR Design}

The optimum design of the suspended CDPR, solution of problem (41), is shown in Fig. 7. The design variables and the objective function values associated with this design are given in Table III. Similarly to the optimum design of the fully constrained CDPR, the distance between the connection points, along $\mathbf{x}_{b}$, are reduced as much as possible, while respecting the static equilibrium constraint. The robot is in static equilibrium thanks to the gravity forces acting on the moving platform. To balance any external wrenches belonging to $[\mathbf{x}]_{r}$ it would be necessary to increase the distance between points $A_{i}$ along the axis $\mathbf{x}_{b}$, possibly generating some collisions between the cables and the structure. 
TABLE III

DESIGN VARIABLE AND OBJECTIVE FUNCTION VALUES OF THE OPTIMUM SUSPENDED CDPR

\begin{tabular}{llllllll}
\hline \hline$h_{p}$ & $l_{p}$ & $w_{p}$ & $u_{x 1}$ & $u_{x 2}$ & $u_{y}$ & $u_{z}$ & $\mathscr{V}$ \\
\hline $0.3 \mathrm{~m}$ & $0.3 \mathrm{~m}$ & $0.6 \mathrm{~m}$ & $0.9 \mathrm{~m}$ & $0.7 \mathrm{~m}$ & $4.363 \mathrm{~m}$ & $4.701 \mathrm{~m}$ & $16.408 \mathrm{~m}^{3}$ \\
\hline \hline
\end{tabular}

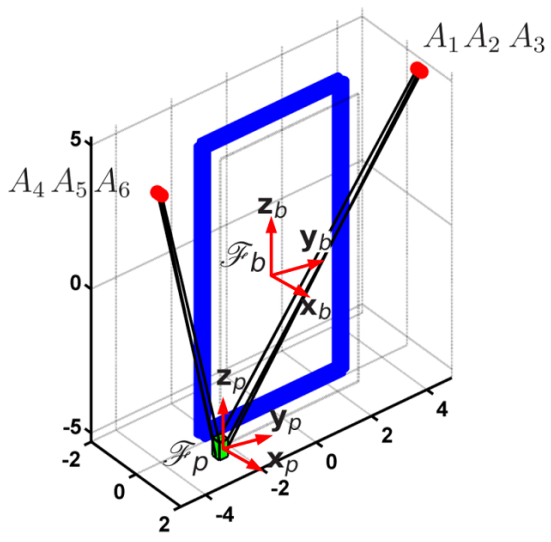

Fig. 7. Optimum design of the suspended CDPR. Solution of the optimization problem (41).

\section{CONCLUSIONS}

This paper introduced a preliminary design study of CDPRs intended to displace painting and sandblasting tools around jackets. This preliminary study considers a simplified four-tube structure in place of a jacket.

The design optimization problem has been formulated for a fully constrained CDPR considering the Cartesian coordinates of points $A_{i}$ as design variables. A suspended CDPR has been studied as well. The minimization of the size of the CDPRs was the objective of both the optimization problems. The size of the optimum fully constrained CDPR is smaller than the size of the optimum suspended CDPR. In the fully constrained CDPR, the connection points are very close to each other along the axis $\mathrm{x}_{b}$. The optimum fully constrained CDPR can assure the static equilibrium of the robot in presence of relevant external wrenches, along the $\mathbf{x}_{b}$ and $\mathbf{y}_{b}$ axes. However, the fully constrained CDPR requires a higher number of cables than the suspended CDPR. Furthermore, the suspended design frees the space below the platform from cables. Overall, if the presence of cable connected to the ground does not generate any issue, the fully constrained solution should be preferred.

The results presented in this paper could be applied to the design of a CDPR painting a single jacket side. The painting and sandblasting of a full jacket may require some reconfigurabilities. The study of reconfigurable CDPRs able to work around a jacket is part of our future work.

\section{ACKNOWLEDGMENT}

This research work has been supported by the IRT Jules Verne, Bouguenais, France (Project CAROCA, Evaluation des CApacités de la RObotique à CÂbles dans un contexte industriel).

\section{REFERENCES}

[1] M. Rock and L. Parsons, "Offshore wind energy," EESI Fact Sheet, Washington, 2010.

[2] J. Albus, R. Bostelman, and N. Dagalakis, "The NIST spider, a robot crane," J. of Research of the Nat. Inst. of Standards and Technology, vol. 97, no. 3, pp. 373-385, May 1992.

[3] C. Holland and D. Cannon, "Cable array robot for material handling," U.S. Patent 6826452, March 2002.

[4] CableBOT [Online]. Available: http://www.cablebot.eu/en/.

[5] SkyCam [Online]. Available: http://skycam.tv/.

[6] SpyderCam [Online]. Available: http://spydercam.com/.

[7] J.-P. Merlet, "Kinematics of the wire-driven parallel robot marionet using linear actuators," in Proc. IEEE Int. Conf. on Robotics and Automation, Passadena, May, pp. 3857-3862.

[8] R. Roberts, T. Graham, and T. Lippitt, "On the inverse kinematics, statics, and fault tolerance of cable-suspended robots," J. of Robotic Systems, vol. 15, no. 10, pp. 581-597, 1998.

[9] P. Bosscher, A. Riechel, and I. Ebert-Uphoff, "rench-feasible workspace generation for cable-driven robots," IEEE Trans. on Robotics, vol. 22, no. 5, pp. 890-902, Oct. 2006.

[10] M. Gouttefarde, D. Daney, and J.-P. Merlet, "Interval-analysis-based determination of the wrench-feasible workspace of parallel cabledriven robots," IEEE Transactions on Robotics, vol. 27, no. 1, pp $1-13$, Feb. 2011

[11] F. Guay, P. Cardou, A. Cruz, and S. Caro, "Measuring how well a structure supports varying external wrenches," in The Second Conference on Mechanisms, Transmissions and Applications, Bilbao, Spain, Oct. 2013.

[12] S. Bouchard, C. M. Gosselin, and B. Moore, "On the ability of a cable-driven robot to generate a prescribed set of wrenches," in Proceedings of the ASME 2008 International Design Engineering Technical Conferences \& Computers and Information in Engineering Conference, Brooklyn, NY, Aug. 2008, pp. 1-12.

[13] M. Gouttefarde and S. Krut, "Characterization of parallel manipulator available wrench set facets," in Advances in Robot Kinematics: Motion in Man and Machine, Piran, 2010, pp. 475-484.

[14] V. Lumelsky, "On fast computation of distance between line segments," Information Processing Letters, vol. 21, 1985.

[15] S. Bezhadipour and A. Khajepour, "Stiffness of cable-based parallel manipulators with application to stability analysis," J. Mechanical Design, vol. 128, pp. 303-310, 2006.

[16] Z. Ugray, L. Lasdon, J. Plummer, F. Glover, J. Kelly, and R. Marti, "Scatter search and local nlp solvers: A multistart framework for global optimization," INFORMS J. on Computing, vol. 19, no. 3, pp. 328-340, 2007. 